UDC 811.111'37

$81^{\prime} 1: 165$

\author{
Marek Kuźniak \\ University of Wrocław, Poland
}

\title{
SOME CRITICAL REMARKS ON ASPECT AS A SITUATION TYPE
}

\begin{abstract}
This paper discusses some controversies that arise in the treatment of aspect as viewed from the perspective of cognitive linguistics ${ }^{1}$. More specifically, the paper constitutes a critical review of Radden and Dirven's (2007) account of aspect as a situation type presented in Chapter 8 of the book. The basic criticism of the category of aspect viewed as a situational type involves, as the author of this paper maintains, unsubstantiated proliferation of the typology of aspectual distinctions, which are hard to account for once the criterion of psychological reality of a conceptual category is considered ${ }^{2}$.
\end{abstract}

\subsection{Fundamentals}

In order to understand how the notion of situation is discussed in cognitive linguistics, let us look at Fig. 1 below.

Thus, the concept of aspect relates conceptually to different types of "time schemas" as indicative of different types of situations. According to Radden and Dirven (2007: 176), there are two types of situation that can be referred to as events and states. The former is illustrative of John wrote several letters, whereas the latter is exemplified by We just like this place as it is. The event differs from the state in that it has a well delineated

\footnotetext{
${ }^{1}$ Radden and Dirven (2007: 198) quote Vendler (1967), Dowty (1979), and Langacker (2001) as representing significant contributions to the study of situation types (the former two books) and introducing into the literature of the subject matter the conception of "basic aspectual class" (the latter). Both insights constitute the foundation of the framework discussed by Radden and Dirven.

${ }^{2}$ See Laurence and Margolis (1999).
} 


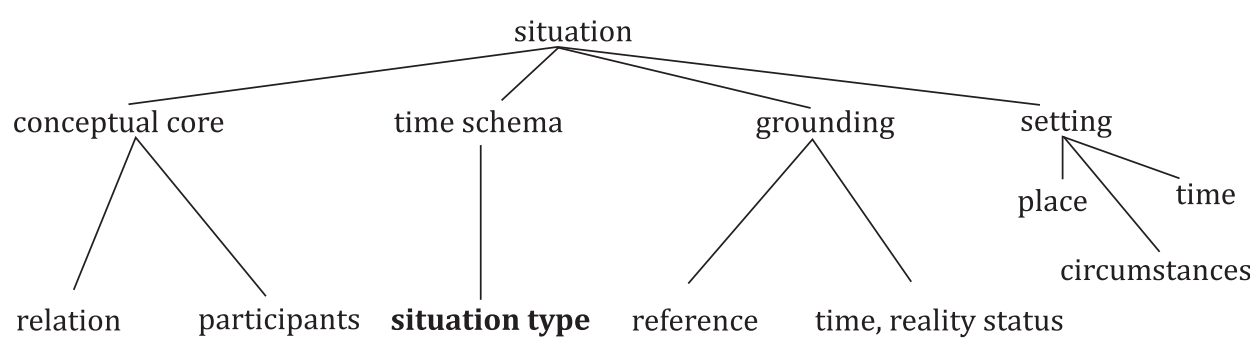

Fig. 1 Elements constituting a situation (after Radden and Dirven 2007: 176)

temporal structure with its starting point and end-point as well as some duration period between the initial and final stage. The state does not exhibit any of the above properties in that it has no starting point, no endpoint, no duration time clearly marked (Radden and Dirven 2007: 176). The authors thus insist that situation types are qualitatively different from other elements constituting a situation in that they have a particular temporal structure. At the same time Radden and Dirven include into the aspectual class types of situation that have atemporal characteristics. This is clearly a contradiction in terms that can only be resolved if we postulate a temporal structure to be manifested in states as well. When we say We just like this place as it is, we implicate the existence of some "earlier time space" in which our attitude to the place was at least neutral, as it takes some time for a human being to get the liking of a particular place. Developing such affection does not happen instantaneously, but usually evolves over a particular stretch of time. So we clearly have in the case of states two stages that are claimed by Radden and Dirven to be missing, i.e. a starting point and a duration period.

Let us move on to the discussion of the fundamental typological distinctions of aspect. Radden and Dirven (2007: 176-177) discuss basic aspectual classes: the non-progressive aspect and the progressive aspect. The former is characterized by "a maximal viewing frame", whereas the progressive is characterized by a "restricted viewing frame". It is to be remembered that a maximal viewing frame enables a human conceptualizer to view the situation in its entirety, whereas the restricted viewing frame highlights but a part of the entire event. This division helps us categorize the following sentences into four different groups which result from the acceptance of the two pairs of variables: event/state and maximal viewing frame/restricted viewing frame. 
a. Ann cuddled the baby (event: maximal viewing frame)

b. Ann is cuddling the baby (event: restricted viewing frame)

c. Ann lives with her parents (state: maximal viewing frame)

d. Ann is living with her parents (state: restricted viewing frame)

(Radden and Dirven 2007: 177)

The descriptions of aspectual meaning pertinent to $a$ and $b$ are rather inarguable. In the case of $a$ we view the situation as a whole, with its beginning, duration, and end-point. In the case of $b$ we concentrate on the internal "durational" aspect of an event. However, due to our everyday experience we are likely to conclude that the boundaries in $b$ are definitely implied as these sorts of situations do not last forever. The problem begins with the explication of $c$. Radden and Dirven (2007: 177) say that the sentence "provides an infinite view of an inherently unbounded and homogeneous or lasting state". What is highly controversial in the above-quoted statement is the category of "inherent unboundedness" as speculated for this situation type. There is no explication provided as to the meaning of "inherent unboundedness" and how such inherent structural types of situation emerge in language. Moreover, there is no conclusive piece of argument in favour of postulating inherently unbounded structure in $c$. To my view, situation $c$ is in no way ontologically different from situations $a$ or $b$, but only epistemically. Thus, $c$ relates to a static kind of event with implicit boundaries, whereas $a$ relates to a dynamic kind of event with explicitly marked boundaries. Sentence $b$ differs from $c$ in that the event is dynamic. However, as it turns out, both $b$ and $c$ have implicitly marked boundaries since both can be classified as events with varying degrees of "boundary designation" within their scope of predication.

Fundamentally, then, the division of situation types into two sets, i.e. states and events fails as it is not compatible with the definition of situation type as manifesting temporal structure. Our conclusion is that the only way we may "exculpate" this understanding of temporal structures is by postulating an ontologically homogeneous type of situation, called "event" which may however undergo modulations involving the selection of either of two distinct ways of viewing, whether maximal or restricted. Regardless of the nature (idiosyncrasy of meaning) of the verb, the "event" may be characterised by two fundamental properties, i.e. dynamicity and state. In light of the criticism spelled out above, the postulated typological distinctions are suggested as follows: 
a. Ann cuddled the baby (event: maximal viewing frame/dynamic)

b. Ann is cuddling the baby (event: restricted viewing frame/ dynamic)

c. Ann lives with her parents (event: maximal viewing frame/static)

d. Ann is living with her parents (event: restricted viewing frame/ static)

The advantage of this typology is that it dismisses the often impressionistic categorial differentiation between an event and a state in favouring one heterogeneous category of event as governed by two qualitatively different sets of viewing perspectives. Fig. 2 below presents a modified version of typology presented by Radden and Dirven (2007):

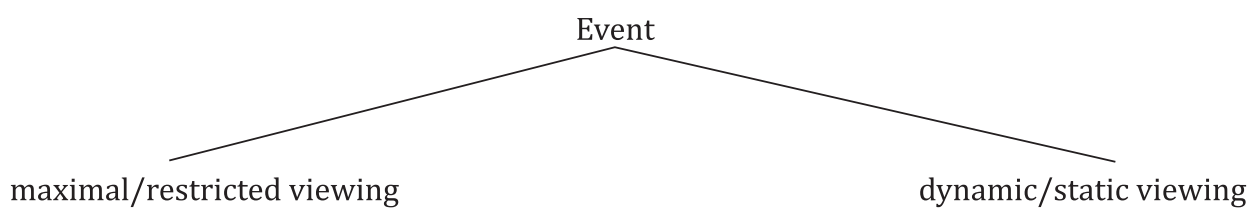

Fig. 2 A modified typology of aspect

Also problematic is the very definition of aspect provided by Radden and Dirven (2007: 176), who classify "aspect as the grammatical form used by a speaker in taking particular view of the situation". As we will show in the subsequent sections of this paper, this definition is too narrow on a cognitive-linguistic account, as it limits the discussion to a set of formal exponents constituting the category of aspect. As the discussion proceeds, we will observe that the category of "aspect" may have some formal manifestations, but quintessentially is to be seen as a cognitive phenomenon whose realisations in language are not only strictly grammatical, but also encoded in the context of situation or conventional semantic load of particular lexical units selected to designate a particular event.

\subsection{Specificities}

The methodological problems arising from postulating the existence of two fundamental kinds of situation, i.e. events and states have got their longlasting consequence on the details of typological organization of situation types as laid down by Radden and Dirven. Events, for example, are further 
subdivided into bounded and unbounded events. This distinction is, as already indicated above, viewed as artificial because we should rather concentrate on the degree of boundedness that a particular event profiles in language than postulate ontologically separate kinds of bounded and unbounded events. The parameters of duration and telicity that cut across the typology presented by Radden and Dirven (2007: 180-181) can be preserved as long as we treat them as non-discrete categories. Thus duration according to the authors (2007: 179) refers to the length of time in which a particular event is seen to occur. In effect we may distinguish between events that occur within a longer stretch of time (display the feature "durational') and those which are short enough to be perceived as long-lasting and therefore are classified as bearing the feature "punctual". Telicity as another parameter is about the possession by an entity of a conclusive (definitive) end-point or the lack of it. Radden and Dirven thus postulate the following typology of events (see Fig. 3-4).

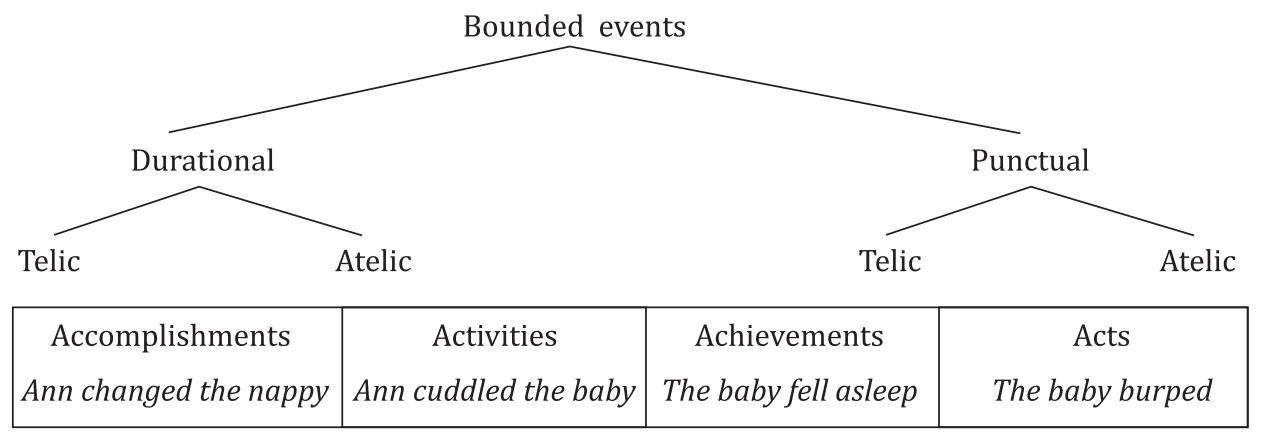

Fig. 3 Bounded events and their classification (after Raden and Dirven 2007: 180)

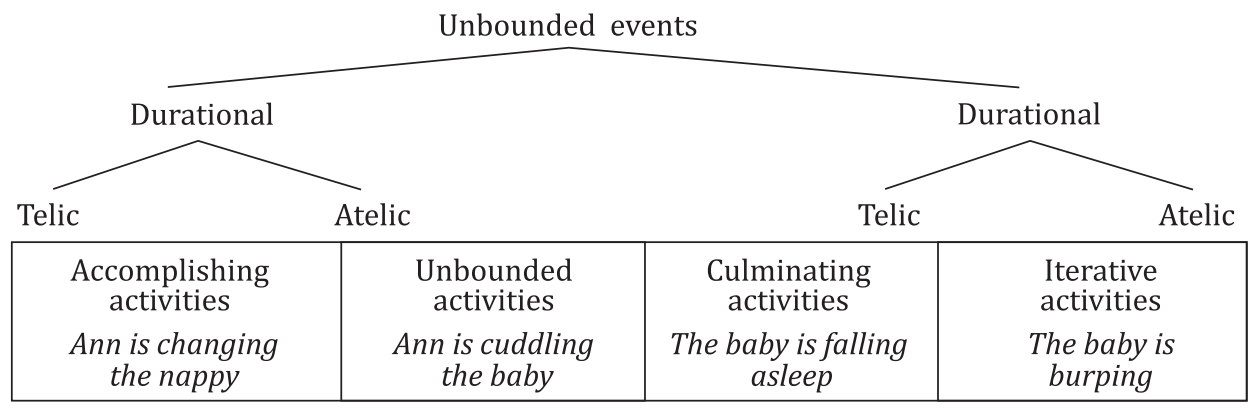

Fig. 4 Unbounded events and their classification (after Radden and Dirven 2007: 181) 
The argument advanced in the present paper is that the above-presented typology, although clear and lucid, unnecessarily abounds in terms whose existence is motivated not so much by perceptual (psychological) criteria of discrimination between categories, but rather is dependent on minute semantic differences between the verbs selected for the analysis, which becomes the source of terminological diversification offered by the book. At the same time, it must be remarked that the choice of some verbs which are meant to illustrate a particular category appears far from fortunate. For example, the verb "burp" affords access to encyclopaedic knowledge structures wherein the activity can hardly be classified as atelic, at least not prototypically. Setting aside the considerations over lexical choices made by Radden and Dirven (2007: 181), we conclude that the category of aspect is no longer correlated to any formal exponency, but rather is conveyed through meaning subtleties encoded by the conventional knowledge interpretation evoked by a particular verb.

The proposal offered in the present paper is to modify the classification illustrated above and integrate Fig. 3 and Fig. 4 into one representation in which the fuzziness of boundedness of the category of event will explicitly be marked.

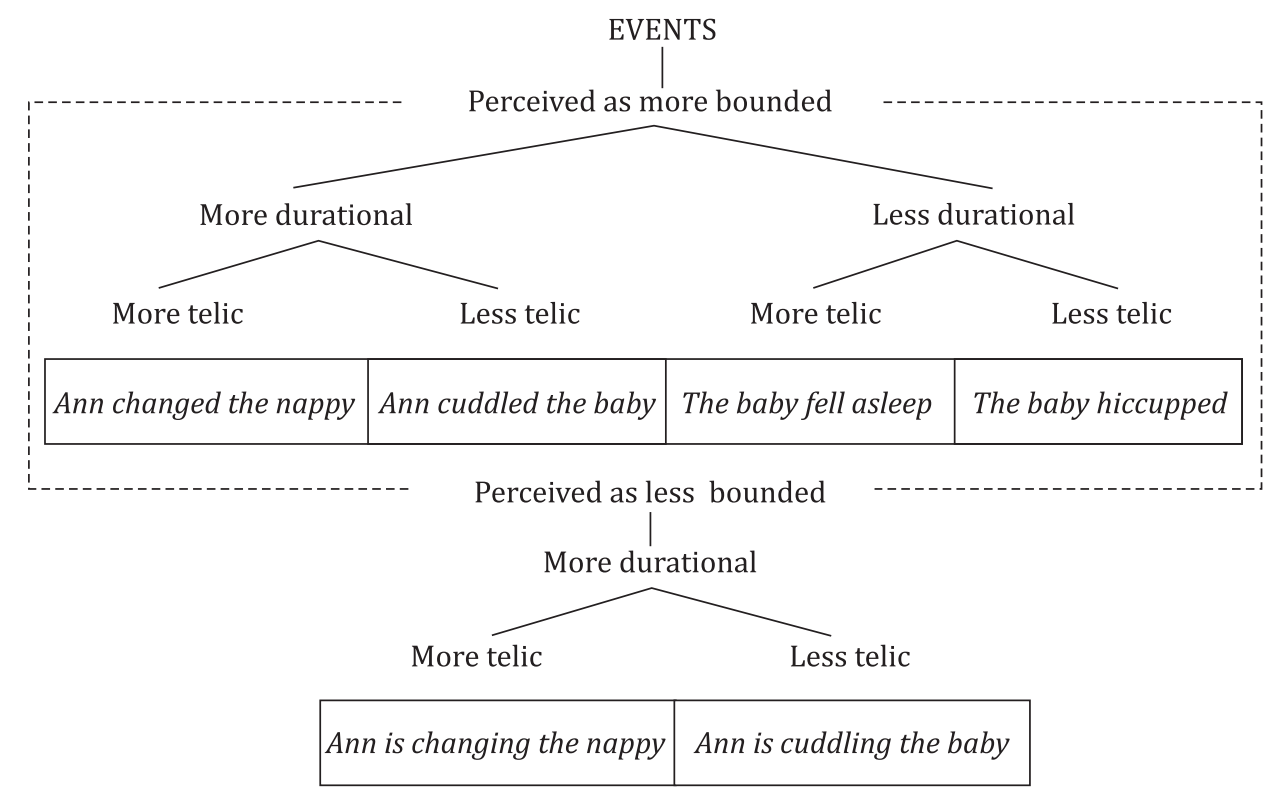

Fig. 5 Events and their classification 
The advantage of such modified typology is that it clearly manifests a continuum-like character of categories relevant to the analysis. This continuum perspective allows us to substantially reduce the number of notions characterising different types of events. Because events are prototypically viewed as CONTAINERS (see Krzeszowski 1997), we therefore assume that the category of boundedness is more psychologically real than this of unboundedness, hence the postulated gradation of boundedness in the above-presented typology. The same holds for the category of telicity. In this case, we resign from the notion of atelicity as prototypical events that are correlated to the SOURCE-PATH-GOAL schema (see Krzeszowski 1997), which constitutes a benchmark for designating less prototypical instances as consistently less GOAL oriented than others. Fig. 1 also appears to get out from the "shackles' of structuralist binarism in the representation of linguistic phenomena. This binary imperative led Radden and Dirven to postulate two nodes branching off the superordinate category of unbounded event in Fig. 4, which are, however, qualitatively the same. We believe that such neat binary typological representations are not the value in themselves in cognitive linguistics, which on the other hand tries to reflect how things are in the world rather than how they should be. Finally, the verb "burp" has been replaced by the verb "hiccup" because it is more in compliance with our everyday experience of this activity as more iterative in character.

As the analysis of aspect continues, Radden and Dirven notice what constitutes quite a considerable departure from their original definition of aspect as a grammatical form, namely they observe that a situation type is not solely a matter of aspect viewed as a grammatical form, nor is it exclusively connected with the semantics of the verb, but rather it is the nature of the situation as a whole that may determine how it is viewed by the human conceptualizer. The conclusion is that the category of aspect is not to be bound with a set of formal correlatives in language, but rather makes up a more dynamically organized category. Such conceived aspect transcends formal exponents of category status and extends over to encapsulate within its scope other lexical elements, e.g. adverbials. This may be exemplified by the following:

a) He sang three songs.

b) He sang three songs all evening.

On a traditional account (see e.g. Quirk and Greenbaum 1973) ${ }^{3}$, these two sentences display the same, so-called simple aspect. We notice,

\footnotetext{
${ }^{3}$ See also classic works by Leech (1971); Palmer (1988) or Comrie (1976).
} 
however, and we do it on experiential grounds, that the addition of an adverbial of time, i.e. all evening, changes the internal viewing structure of an event from less durational and at the same time more telic (sentence a)) to a more durational and less telic (sentence b)). All in all, my argument goes like this: we can discuss all the slightest differences in aspectual organization of an event without recourse to multiplying different categories like "accomplishments", "accomplishing activities", "achievements", "acts", etc., because such proliferation of labels induces the reader to search for clear-cut discriminative criteria where fuzziness seems to prevail.

This methodological imperative for the search of different names for particular categories of events causes Radden and Dirven, aside from the aforementioned pitfalls, to sound rather inconclusive when it comes to finding more elaborate explications of multifarious differences encoded by the use of progressive or non-progressive forms ${ }^{4}$. Paradoxically, these explications indirectly corroborate what has been clearly stated in this paper, i.e. that the category of aspect - a dynamically organized category - is assigned the details of internal event viewing framework online, i.e. via the complex process of contextual information retrieval. This certainly does not exclude the assumption that progressive and non-progressive forms do not have conventional, schematic interpretations that humans impose on viewing the structure of an event. But given the validity of the existence of such conventionally encoded interpretative frameworks, they can only be reduced to general conceptual categories of duration, boundedness and telicity as these constitute a framework of aspectual organization of any event described in language.

\subsection{Conclusion}

Thought-provoking and insightful as Radden and Dirven's (2007) cognitive account of the category of aspect may be, it appears to unnecessarily go into detailed binary typological classifications, which otherwise can perfectly be

\footnotetext{
${ }^{4}$ Such is the case when Radden and Dirven (2007: 187) attempt to discuss the difference between the following two sentences: a) I talked to Mr. Green, and b) I was talking to Mr. Green. In a) the non-progressive form according to Radden and Dirven "invites implicatures of factuality and determination" whereas in b) suggests that "I possibly happened to meet Mr Green, that we talked for talk's sake, and that we only had some casual small talk" (Radden and Dirven 2007: 187). Such detailed interpretations that hinge upon the notions of other than those strictly temporal cannot, in my view, be easily captured within a conventional implicature encoded by these forms, but rather are a function of complex processes of contextual modulation of information (see Cruse 1986) during which rich encyclopaedic knowledge structures are activated.
} 
handled through a recourse to a few conceptual schematic categories like boundedness, duration, or telicity. An important reservation is that these categories are construed along a certain continuum, rather than neatly pigeon-holed into well-defined fields. This is far from a revolutionary statement, but rather a simple restatement of one of the fundamental tenets behind a cognitive-linguistic enterprise which postulates fuzziness of category boundaries in natural language (see Rosch 1977, Evans and Green 2006). Of course, we do not claim that Radden and Dirven's account ignores the aspect of fuzziness whatsoever. However, because they lay the emphasis on introducing specific typological distinctions as different manifestations of event viewing frames, they appear to defocus, at the same time, on the factor of non-discreteness of categories, which leads to a certain degree of fallacy behind the argumentation advanced by the eminent scholars.

The best illustration of such fundamental fallacy is an attempt at explicating the category of "culminating activity", which Radden and Dirven (2007: 188) themselves acknowledge "for the lack of a better term" (sic!), as a special type of an unbounded event ${ }^{5}$. The motivation for coining the label comes from the assumptions made at the outset (see Fig. 3) where the category of "achievements" was postulated as inherently bounded (He fell asleep), so Radden and Dirven did not admit of any unbounded versions of this category of event. They, on the other hand, state somewhat straightforwardly: "There are no unbounded achievements since achievements cannot be extended in time". This led them to search for a different label that would not distort once accepted typology. Such label was found and the event was finally categorized as "culminating activity". The sole reason behind the rejection of the term "unbounded achievement" was thus stylistic (a matter of labelling) rather than motivated by other cognitively relevant factors.

Last but not least, we may hypothesize that the ultimate source of various problems in providing conclusive explications of aspectual organization of events by Radden and Dirven consists in the illegitimate ${ }^{5}$ The category of culminating activity is instantiated by the following: The Queen is arriving
and prompted for by the illustration (after Radden and Dirven 2007: 189).

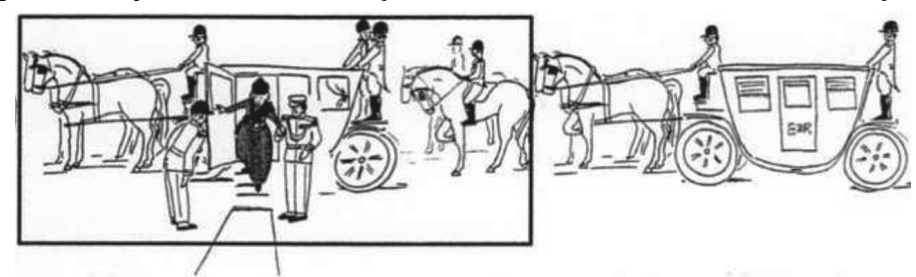


interchangeability of qualitatively different levels of description in which a schematic definitive argumentative formula $x$ is $y$ is seen as synonymous with a tentative argumentative formula $x$ is perceived as. These, in my view, introduce two ontologically separate modes of existence; therefore, they cannot be so freely interchanged. Taking the aforementioned example of The Queen was arriving, we notice that Radden and Dirven use the formula $x$ is $y$ in postulating the necessity of naming the event as a "culminating activity" rather than "unbounded achievement", because as they claim "unbounded achievements cannot be extended in time". So their definition of unbounded events is quite definitive. At the same time they show that once we find a different label for the same event (e.g. culminating activity), it is perfectly possible to view the same event as extendable temporally due to the working of conceptual metonymies UNIPLEX FOR MULTIPLES and TERMINAL POINT FOR ACTIVITY. Such loose treatment of the two formulas in substantiating the stylistic aspect of finding a name with reference to the same event should be seen as rather a risky enterprise.

\section{References}

Comrie, B. (1976). Aspect. Cambridge: Cambridge University Press.

Cruse, D. A. (1986). Lexical Semantics. Cambridge: Cambridge University Press.

Dowty, D. R. (1979). Word Meaning and Montague Grammar: The Semantics of Verbs and Times in Generative Semantics and in Montague's PTQ. Dordrecht: Reidel.

Evans, V. and M. Green (2006). Cognitive Linguistics. An Introduction. Edinburgh: Edinburgh University Press.

Krzeszowski, T. P. (1997). Angels and Devils in Hell. Warszawa: Wydawnictwo Energeia.

Langacker, R. (2001). The English present tense. English Language and Linguistics 5: 251-272.

Laurence, S. and M. Margolis (1999). Concepts and cognitive science. In: E. Margolis and S. Laurence (eds.), Concepts: Core Readings. Cambridge, MA: MIT Press. 3-81.

Leech, G. (1971). Meaning and the English Verb. London: Longman.

Palmer, F. (1988). The English Verb. $2^{\text {nd }}$ ed. London: Longman.

Quirk, R. and S. Greenbaum (1973). The University Grammar of English. London: Longman. 
Radden, G. and R. Dirven (2007). Cognitive English Grammar. Amsterdam/ Philadelphia: John Benjamins Publishing Company.

Rosch, E. (1977). Human categorization. In: N. Warren (ed.), Studies in Cross-linguistic Psychology. London: Academic Press, 1-49.

Vendler, Z. (1967). Linguistics in Philosophy. Ithaca NY: Cornell University Press. 
